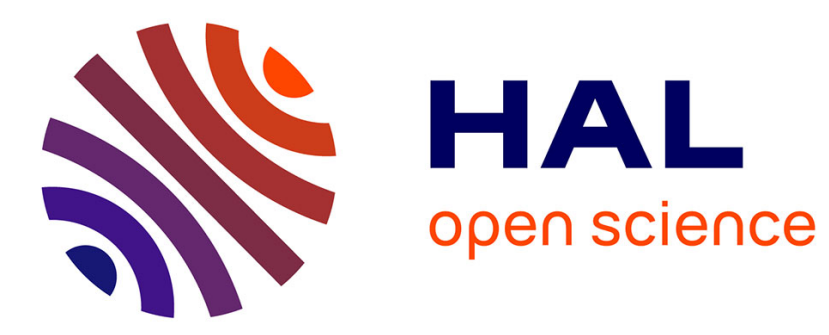

\title{
Enablers for high order level set methods in fluid mechanics
}

Francky Luddens, Michel Bergmann, Lisl Weynans

\section{To cite this version:}

Francky Luddens, Michel Bergmann, Lisl Weynans. Enablers for high order level set methods in fluid mechanics. [Research Report] RR-8656, INRIA Bordeaux; INRIA. 2014. hal-01097185

\section{HAL Id: hal-01097185 \\ https://hal.inria.fr/hal-01097185}

Submitted on 19 Dec 2014

HAL is a multi-disciplinary open access archive for the deposit and dissemination of scientific research documents, whether they are published or not. The documents may come from teaching and research institutions in France or abroad, or from public or private research centers.
L'archive ouverte pluridisciplinaire HAL, est destinée au dépôt et à la diffusion de documents scientifiques de niveau recherche, publiés ou non, émanant des établissements d'enseignement et de recherche français ou étrangers, des laboratoires publics ou privés. 


\title{
I N R I A
}

INSTITUT NATIONAL DE RECHERCHE EN INFORMATIQUE ET EN AUTOMATIQUE

\section{Enablers for high order level set methods in fluid} mechanics

\author{
Francky Luddens — Michel Bergmann — Lisl Weynans
}

$\mathrm{N}^{\circ} 8656$

Nov. 2014

\section{apport}

de recherche 



\title{
Enablers for high order level set methods in fluid mechanics
}

\author{
Francky Luddens*, Michel Bergmann ${ }^{\dagger}$, Lisl Weynans ${ }^{\ddagger}$ \\ Theme : \\ Équipe-Projet MC2
}

Rapport de recherche $\mathrm{n}^{\circ} 8656$ - Nov. 2014 - 22 pages

\begin{abstract}
In the context of numerical simulations of multiphysics flows, accurate tracking of an interface and consistent computation of its geometric properties are crucial. In this paper, we investigate a level set technique that satisfies these requirements, even for long times computations. The method is developed in a finite differences framework on cartesian grids. As in usual level set strategies, reinitialization steps are involved. Several reinitialization algorithms are reviewed and mixed to design an accurate and fast reinitialization procedure. When coupled with a time evolution of the interface, the reinitialization procedure is performed only when there are too large deformations of the isocontours. This strategy limits the number of reinitialization steps and shows a good balance between accuracy and computational cost. Numerical results compare well with usual level set strategies and confirm the necessity of the reinitialization procedure, together with a limited number of reinitialization steps.
\end{abstract}

Key-words: level set; redistanciation; Eikonal equation; finite differences; two-phase flows

* INRIA Bordeaux Sud-Ouest, Equipe MC2

$\dagger$ INRIA Bordeaux Sud-Ouest, Equipe MC2

‡ INRIA Bordeaux Sud-Ouest, Equipe MC2 


\section{Méthodes level set d'ordre élevé en mécanique des fluides}

Résumé : Lors de la simulation d'écoulements multiphysiques, l'un des enjeux majeurs est le suivi précis des interfaces, ainsi que le calcul de leurs propriétés géométriques. Dans ce rapport, nous nous intéressons à une approche level set permettant la localisation et le calcul précis de ces propriétés, même pour des simulations en temps long. La méthode employée repose sur des diffréences finies sur grille cartésienne. Une procédure de réinitialisation est employée, pour maintenir la level set proche d'une fonction distance. Plusieurs méthodes standards de réinitialisation sont étudiées et couplées pour obtenir une procédure rapide et précise. Pour des interfaces évoluant avec le temps, cette phase de réinitialisation n'est utilisée que lorsque la déformation des iso contours de la level set est trop importante. Cette stratégie permet de limiter le nombre de réinitialisations, et offre un bon compromis entre précision et coût de calcul. Des illustrations numériques montrent le bon comportement de cette méthode vis à vis de méthodes level set standards; elles confirment également la nécessité de la phase de réinitialisation, ainsi que la nécessité de contrôler le nombre de réinitialisations.

Mots-clés : méthode level set; redistanciation; équation eikonale; différences finies; fluides diphasiques 


\section{Introduction}

In multiphysics flows (multiphase flows, fluid-solid interactions), it is essential to be able to accurately track and represent the evolution of interfaces. For example, in a Lagrangian framework, particle methods (such as front tracking algorithms [22]) or conforming meshes [13], might be used. These techniques allow a very accurate localization of the interface, but might require possibly complex reseeding or remeshing algorithms, especially for large deformations of the interface. They can be mixed with Eulerian methods with the ALE method, introduced by Hirt et al. [9]. In an Eulerian framework, an efficient way of capturing interfaces is the level set method, introduced by Osher and Sethian [14]. It consists of the capture of an interface through the 0-isocontour of a higher dimensional function. This eases the treatment of topological changes and can also easily be carried on in parallel codes, using domain decomposition for example.

In several applications, an accurate computation of the geometric properties of the interface is required: for example, the computation of the normal to the interface for methods with second order penalty terms in fluid-solid interactions [2] or for computations on numerical domains that do not fit the physical domain [7]. For the cases of multiphysics flows where the surface tension plays an important role, accurate computation of the curvature is a key stone. In general, distortions of the level set function $\phi$ might lead to significant errors in computing the geometric properties. One possible way to avoid large errors is the use of the distance function instead of a generic level set function. Unfortunately, even with a distance function as an initial state, $\phi$ can derive far from the distance function. To circumvent the problem, Sussman et al. [20] introduced a reinitialization algorithm; it consists of an algorithm capable of recomputing the distance function. The current level set function is then replaced by this simpler function for the advection part. The reinitialization procedure may also be used in the context of the CSF method developed by Brackbill et al.[3], where the interface is diffuse: it allows to keep a constant width for the interface.

Several methods have been developed over the years to perform the reinitialization step. It is based on the resolution of the eikonal equation

$$
|\nabla \phi|=1
$$

either by searching a stationary solution to a time dependent Hamilton-Jacobi equation [20] or using a Gauss-Seidel based method as in fast marching methods [16, 18] or fast sweeping methods [21]. The original method from [20] can be modified to ensure at least first order accuracy on the curvature of the interface, but it is CPU consuming. The fast marching or fast sweeping methods are on the contrary much quicker, but might suffer from a lack of accuracy.

The usual level set strategy for an evolving interface is the following:

- Use a transport equation to update $\phi$,

- From time to time, reinitialize $\phi$ with the signed distance function: this step is useful for geometric properties computation, and it also eases the transport step, since the advected function is simpler and smoother.

Although the reinitialization procedure may improve mass conservation [20], it also introduces some error by slightly moving the interface [17]. It is then unclear what the frequency of these reinitialization steps should be.

In this paper, we investigate a (not too expensive) level set technique that ensures a good representation of the interface, as well as its geometrical properties, even for long times. The method is developped in a finite differences framework on cartesian grids. It has been developped in such a way that parallization should be easily and efficiently available. The strategy is based on a limited number of reinitialization steps. These steps are chosen according to the deformation of the isocontours of the level set function. They are necessary to prevent creation of small and/or large gradients near the interface, which could lead to numerical instabilities or increasing error on geometric computations. 
The paper is organized as follows: In $\S 2$ we present the framework and the objectives we want to reach using a level set technique. In $\S 3$, we discuss high order reinitialization schemes, and we design a method with a balance between accuracy and computational cost. The strategy for the coupling transport/reinitialization is discussed in $\S 4$. We conclude in $\S 5$ with a discussion about the numerical results we obtained.

\section{Motivations}

\subsection{Framework}

Let $\Omega=\left[x_{\min } ; x_{\max }\right] \times\left[y_{\min } ; y_{\max }\right]$ be a two dimensional rectangular domain in which we want to track the evolution of an interface $\Gamma$. Let us denote by $\Gamma_{0}$ the initial interface. We choose to capture $\Gamma$ through the zero isocontour of a level set function $\phi . \boldsymbol{U}(\boldsymbol{x}, t)$ denotes the velocity field for the advection of $\Gamma$. In order to mimic multiphysics flows, we assume that $\boldsymbol{U}$ is available in the whole domain $\Omega$, either with explicit expressions or obtained by separate computations. Since we are mainly interested in incompressible fluid mechanics, we will often use divergence free velocity fields. In this context, the evolution of $\Gamma$ is recovered from the evolution of $\phi$, that satisfies the non conservative equation

$$
\begin{aligned}
\partial_{t} \phi+\boldsymbol{U} \cdot \nabla \phi & =0, \\
\phi_{\mid t=0} & =d_{0},
\end{aligned}
$$

or the conservative form, when $\nabla \cdot \boldsymbol{U}=0$,

$$
\begin{aligned}
\partial_{t} \phi+\nabla \cdot(\phi \boldsymbol{U}) & =0, \\
\phi_{\mid t=0} & =d_{0},
\end{aligned}
$$

where $d_{0}$ is the signed distance function to $\Gamma_{0}$. Similarly, we will use the notation $d$ for the signed distance to $\Gamma$. Finally, we denote by $\boldsymbol{n}$ (resp. $\kappa$ ) the normal vector (resp. curvature) on the interface $\Gamma$.

The governing equations are discretized using a cartesian mesh: we denote by $N_{x}$ and $N_{y}$ the number of nodes in each direction. The mesh size in each direction is defined by

$$
\Delta x=\frac{x_{\max }-x_{\min }}{N_{x}-1}, \quad \Delta y=\frac{y_{\max }-y_{\min }}{N_{y}-1} .
$$

The nodes are denoted by $\boldsymbol{x}_{i, j}:=\left(x_{i}, y_{j}\right)$ for $i=1, \cdots, N_{x}$ and $j=1, \cdots, N_{y}$. We choose a uniform spacing for the nodes, i.e.,

$$
x_{i}=x_{\min }+(i-1) \Delta x, \quad y_{j}=y_{\min }+(j-1) \Delta y .
$$

For a given field $F$, we obviously denote by $F_{i, j}$ its approximate value at node $\boldsymbol{x}_{i, j}$. For the sake of simplicity, we often assume that $\Delta x=\Delta y$, but the ideas presented here can be carried out with different mesh sizes. We sometimes also denote by $h$ the typical mesh size (i.e., $h=\Delta x$ ).

We work on this cartesian grid in a finite differences framework. We then introduce the notations $D_{x}^{ \pm}$and $D_{y}^{ \pm}$for the one-sided derivatives of a field. The definition of these quantities will differ depending on the equation we are solving and the accuracy we want to reach.

\subsection{Geometric properties computation}

In the level set framework, the computation of the normal and the curvature of $\Gamma$ is straightforward. Recall that the normal $\boldsymbol{n}$ and the curvature $\kappa$ are given by

$$
\boldsymbol{n}:=\frac{\nabla \phi}{|\nabla \phi|}, \quad \kappa:=\nabla \cdot \boldsymbol{n} .
$$


If $\phi$ is the signed distance function, owing to the property $|\nabla \phi|=1$, these expressions read in an even simpler way $\boldsymbol{n}=\nabla \phi$ and $\kappa=\Delta \phi$. Owing to the definitions of $\boldsymbol{n}$ and $\kappa$, we rewrite (2.5) as

$$
\kappa=\frac{\phi_{x}^{2} \phi_{y y}+\phi_{y}^{2} \phi_{x x}-2 \phi_{x} \phi_{y} \phi_{x y}}{\left(\phi_{x}^{2}+\phi_{y}^{2}\right)^{3 / 2}},
$$

where $\phi_{x}$ is the first derivative of $\phi$ with respect to the first variables (and similar definitions for $\phi_{y}, \phi_{x x}, \phi_{x y}$ and $\left.\phi_{y y}\right)$.

For numerical computations, the amplitude of the error depends on the chosen level set function. Let us illustrate this fact on a simple circle of radius $R=0.6$. We consider the following setting:

$$
\begin{aligned}
\Omega & :=[-1 ; 1]^{2}, \\
\Gamma & :=\left\{x^{2}+y^{2}=R^{2}\right\}, \\
d(x, y) & =\sqrt{x^{2}+y^{2}}-R,
\end{aligned}
$$

and we introduce a level set function $\phi_{0}$ that vanishes on $\Gamma$,

$$
\phi_{0}(x, y):=\frac{d(x, y)}{R}\left(\epsilon+\left(x-x_{p}\right)^{2}+\left(y-y_{p}\right)^{2}\right),
$$

with $\epsilon=0.02, x_{p}=0.7$ and $y_{p}=0.4$. We compute the curvature on $\Gamma$ in three different ways:

1. computing $\nabla \cdot\left(\frac{\nabla \phi_{0}}{\left|\nabla \phi_{0}\right|}\right)$, using $(2.6)$,

2. computing $\nabla \cdot\left(\frac{\nabla d}{|\nabla d|}\right)$, using $(2.6)$,

3. computing $\Delta d$.

The last two computations are supposed to be equivalent, but the numerical approximations differ. We use centered second order finite differences formulas to compute the derivatives, i.e.,

$$
\begin{aligned}
\phi_{x, i, j} & =\frac{\phi_{i+1, j}-\phi_{i-1, j}}{2 \Delta x}, & \phi_{y, i, j}=\frac{\phi_{i, j+1}-\phi_{i, j-1}}{2 \Delta y}, \\
\phi_{x x, i, j} & =\frac{\phi_{i+1, j}-2 \phi_{i, j}+\phi_{i-1, j}}{\Delta x^{2}}, & \phi_{y y, i, j}=\frac{\phi_{i, j+1}-2 \phi_{i, j}+\phi_{i, j-1}}{\Delta y^{2}}, \\
\phi_{x y, i, j} & =\frac{\phi_{i+1, j+1}+\phi_{i-1, j-1}-\phi_{i-1, j+1}-\phi_{i+1, j-1}}{4 \Delta x \Delta y} . &
\end{aligned}
$$

The $L^{\infty}$ error on the curvature for different mesh sizes is represented in Figure 1. All three computations exhibit the expected second order accuracy, but the amplitude of the error is much lower in the case of $d$ (reduction by a factor of 100). This example shows that if the level set function has strong and/or weak gradients near the interface, the accuracy of the computation of the curvature is deteriorated. Even though the three computations show a second order convergence, it confirms that it is interesting to work with distance functions, since it provides the lowest amplitude on the error.

The rest of this paper is devoted to the design of a fast level set strategy involving reinitialization steps that satisfies two requirements:

- ensure at least first order accuracy for the computation of $\kappa$,

- provide a small amplitude of the error.

The first step is the design of a fast and accurate reinitialization procedure for static interfaces. The second step is the development of a strategy for the coupling transport/reinitialization, in order to accurately track moving interfaces. The challenge is to carefully control the gradient of the numerical level set function without degrading the accuracy of the numerical solution. 


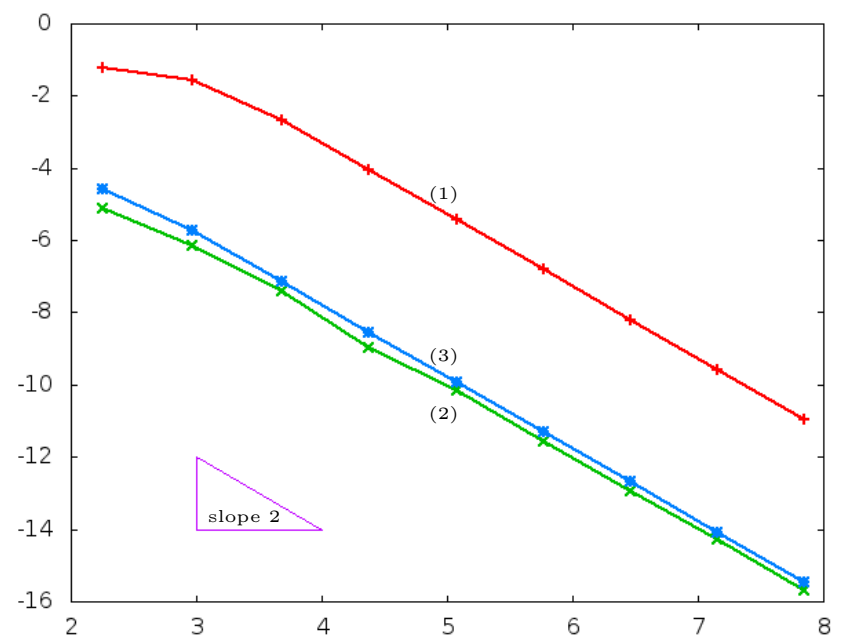

Figure 1: $L^{\infty}$ error on the curvature of the interface vs $1 / \Delta x$ (log-log representation): curvature of the original level set (1), curvature of the distance function (2), laplacian of the distance function $(3)$.

\section{3 high order reinitialization schemes}

In order to ensure numerical stability of the computations, reinitialization procedures are commonly used [20]: it consists in replacing the current level set function by the signed distance function. Given an original level set function $\phi_{0}$, the aim is to find $\phi$ such that the eikonal equation is satisfied, i.e.,

$$
\begin{aligned}
|\nabla \phi| & =1, \\
\phi & =0 \text { on }\left\{\phi_{0}=0\right\} .
\end{aligned}
$$

A high order accuracy near the interface is required to ensure good approximations of the geometric properties. We want at least first order accuracy on $\kappa$, which means that we need at least third order accuracy on $\phi$ (for a classical scheme) near $\Gamma$. Several methods, such as relaxation methods, fast sweeping methods or fast marching methods, have been designed to solve the eikonal equation. We start by reviewing the first two, and develop a mixed method to get a fast reinitialization procedure. We do not use fast marching algorithms since they are not likely to be carried on in parallel codes.

\subsection{Relaxation method}

Relaxation methods are based on the resolution of the unsteady PDE

$$
\partial_{\tau} \phi+\operatorname{sign}\left(\phi_{0}\right)(|\nabla \phi|-1)=0,
$$

where $\tau$ is a fictitious time, until the steady state is reached [20]. This equation is analogous to a non linear transport equation with source term:

$$
\partial_{\tau} \phi+\operatorname{sign}\left(\phi_{0}\right) \frac{\nabla \phi}{|\nabla \phi|} \cdot \nabla \phi=\operatorname{sign}\left(\phi_{0}\right) .
$$

The velocity for the transport is of magnitude 1 , which means that the steady state should be reached for $\tau \sim L_{\max }$ where $L_{\max }$ is the maximum distance between the domain boundary and the interface. This also implies a CFL condition for iteratively solving (3.3), which is $\Delta \tau \leq \Delta x$. This condition implies that the number of iterations needed for convergence is multiplied by 2 


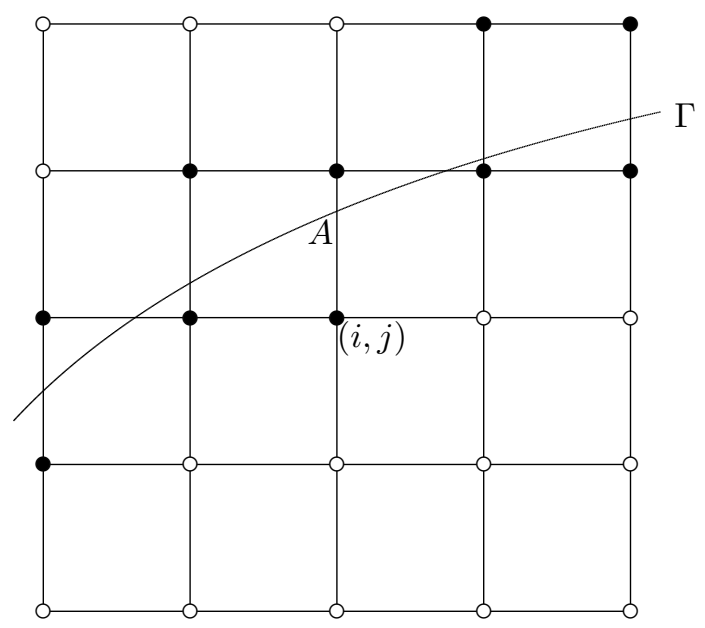

Figure 2: Illustration for the subcell fix. Plain circles stand for nodes close to the interface, empty circles are nodes away from the interface. For example, the stencil used for $D_{y}^{ \pm} \phi_{i, j}$ is $\left(\boldsymbol{x}_{i, j-2} ; \boldsymbol{x}_{i, j-1} ; \boldsymbol{x}_{i, j} ; \boldsymbol{x}_{A} ; \boldsymbol{x}_{i, j+1}\right)$.

when the mesh size is divided by 2 . This method is then a priori rather slow. In practice, we use $\Delta \tau=\Delta x / 2$ and a third order Runge-Kutta TVD scheme for pseudo-time evolution, see [8].

The relaxed eikonal equation (3.3) is a particular case of an Hamilton-Jacobi equation; numerical schemes for such equations might be found in [11]. In our particular case, we rewrite (3.3) in the semi-discrete form

$$
\partial_{\tau} \phi+S\left(\phi_{0}\right) H_{G}\left(D_{x}^{+} \phi, D_{x}^{-} \phi, D_{y}^{+} \phi, D_{y}^{-} \phi\right)=0,
$$

where $H_{G}$ is the Godunov flux defined as

$$
H_{G}(a, b, c, d):= \begin{cases}\sqrt{\max (a,-b, 0)^{2}+\max (c,-d, 0)^{2}} & \text { if } \operatorname{sign}\left(\phi_{0}\right) \leq 0, \\ \sqrt{\max (-a, b, 0)^{2}+\max (-c, d, 0)^{2}} & \text { if } \operatorname{sign}\left(\phi_{0}\right)>0 .\end{cases}
$$

$S\left(\phi_{0}\right)$ is a smoothed sign function, defined by

$$
S\left(\phi_{0}\right)=\frac{\phi_{0}}{\sqrt{\phi_{0}^{2}+\left|\nabla \phi_{0}\right|^{2}(\Delta x)^{2}}} .
$$

The accuracy of the algorithm depends on the computation of the one-sided derivatives $D_{x}^{ \pm} \phi$ and $D_{y}^{ \pm} \phi$ used in the Godunov flux. No matter which method is used for the computation, the scheme slightly moves the interface away from its original position. It has been showed by Russo and Smereka [17] that the use of a standard WENO-5 scheme leads to a displacement that increases with the number of iterations. To improve the accuracy of the method, it is important to be able to limit this displacement. Since most of it comes from the stencil crossing the interface, they introduced the so called subcell fix that ensure the displacement will stop after a certain number of iterations. Using the subcell fix, they obtained a globally second order accurate method. This idea has been carried on in [6] where a third order scheme is developped. The choice of $D_{x}^{ \pm} \phi$ and $D_{y}^{ \pm} \phi$ is as follows:

- if the point is close to the interface (see Figure 2), use a non-uniform stencil with 5 points and an ENO-3 scheme, detailed in [6],

- otherwise, use the standard WENO-5 scheme, see [12]. 


\subsection{Fast sweeping methods}

Fast sweeping methods ([21] for the original method or [23] for high order schemes) are based on Gauss-Seidel like iterations to solve the eikonal equation. The grid is swept in four alternating directions, namely

$$
\begin{aligned}
& \text { (1) } i=1, \cdots n_{x}, j=1, \cdots n_{y}, \\
& \text { (2) } i=n_{x}, \cdots 1, j=1, \cdots n_{y}, \\
& \text { (3) } i=n_{x}, \cdots 1, j=n_{y}, \cdots 1, \\
& \text { (4) } i=1, \cdots n_{x}, j=n_{y}, \cdots 1,
\end{aligned}
$$

and the values of $\phi$ are updated on the fly. The idea is similar to fast marching methods (see [18] e.g. ), but instead of following characteristics flowing from $\Gamma$, the nodes are updated when the characteristics are in the direction of sweeping. The standard first order method, denoted here by fs 1 , to update $\phi_{i, j}$ is the following: freeze all values of $\phi$ and find $\phi_{i, j}^{n e w}$ such that

$$
\left[\left(s\left(\frac{\phi_{i, j}^{n e w}-\phi_{\min }^{x}}{\Delta x}\right)\right)^{+}\right]^{2}+\left[\left(s\left(\frac{\phi_{i, j}^{n e w}-\phi_{\min }^{y}}{\Delta x}\right)\right)^{+}\right]^{2}=1
$$

where $(x)^{+}:=\max (x, 0), s=\operatorname{sign}\left(\phi_{i, j}\right)$ and $\phi_{\min }^{x}$ is defined by

$$
\phi_{\min }^{x}:=s \min \left(s \phi_{i-1, j}, s \phi_{i+1, j}\right) .
$$

$\phi_{\min }^{y}$ is defined in a similar way. This first order scheme is purely upwind and monotone, so that it requires a number of iterations that is independent of the mesh size, see [25]. In [23], higher order methods are achieved, denoted here by fs3z, using

$$
\phi_{\min }^{x}:=s \min \left(s\left(\phi_{i, j}-\Delta x D_{x}^{-} \phi_{i, j}\right) ; s\left(\phi_{i, j}+\Delta x D_{x}^{+} \phi_{i, j}\right)\right),
$$

and a WENO-3 scheme for $D_{x}^{ \pm}$and $D_{y}^{ \pm}$. Unfortunately, it requires a number of iterations that increases with the number of degrees of freedom.

We designed a second order method, fs2, that turned out to have a stable number of iterations, by replacing the expressions in (3.5) by

$$
\left[\left(s\left(\frac{\phi_{i, j}^{n e w}-\phi_{\min }^{x}}{\alpha \Delta x}\right)\right)^{+}\right]^{2}+\left[\left(s\left(\frac{\phi_{i, j}^{n e w}-\phi_{\min }^{y}}{\alpha \Delta x}\right)\right)^{+}\right]^{2}=1
$$

with $\alpha=\frac{2}{3}$ and

$\phi_{\min }^{x}:=\left\{\begin{array}{ll}\frac{4}{3} \phi_{i-1, j}-\frac{1}{3} \phi_{i-2, j} & \text { if } s \phi_{i-1, j} \leq s \phi_{i+1, j} \\ \frac{4}{3} \phi_{i+1, j}-\frac{1}{3} \phi_{i+2, j} & \text { otherwise }\end{array} \quad \phi_{\min }^{y}:=\left\{\begin{array}{ll}\frac{4}{3} \phi_{i, j-1}-\frac{1}{3} \phi_{i, j-2} & \text { if } s \phi_{i, j-1} \leq s \phi_{i, j+1} \\ \frac{4}{3} \phi_{i, j+1}-\frac{1}{3} \phi_{i, j+2} & \text { otherwise }\end{array}\right.\right.$.

The complete fast sweeping scheme is as follows:

- Initialization near $\Gamma$ : set values in the vicinity of the interface, e.g. in the band $B_{n}:=$ $\{d(x, \Gamma) \leq 5 \Delta x\}$. These values remain untouched after.

- Initialization of other values: for the method $\mathrm{fs} 1$, the other values are set to $\pm 10^{8}$ depending on the sign of $d$ [21]. For higher order methods fs 2 and fs3z, we use the first order approximation given by $\mathbf{f} \mathbf{s}$ as the initial state.

- Iteration: update $\phi$ elsewhere by sweeping the grid in four alternating directions,

- Convergence: stop the computation when the $L^{1}$ norm between two successive iterations is small enough. 


\begin{tabular}{|c||c|c|c||c|c|c||c|c|c|}
\hline \multicolumn{1}{|c||}{$\frac{1}{\Delta x}$} & \multicolumn{3}{c||}{ fs1 } & \multicolumn{3}{c||}{ fs3Z } & \multicolumn{3}{c|}{ fs2 } \\
\cline { 2 - 9 } & error & coc & $N_{i t}$ & error & coc & $N_{i t}$ & error & coc & $N_{i t}$ \\
\hline 20 & $1.21 \mathrm{E}-02$ & - & 3 & $3.85 \mathrm{E}-04$ & - & 17 & $1.56 \mathrm{E}-03$ & - & 7 \\
40 & $6.29 \mathrm{E}-03$ & 0.91 & 3 & $5.25 \mathrm{E}-05$ & 2.77 & 28 & $5.44 \mathrm{E}-04$ & 1.47 & 7 \\
80 & $3.18 \mathrm{E}-03$ & 0.97 & 3 & $7.41 \mathrm{E}-06$ & 2.77 & 27 & $1.58 \mathrm{E}-04$ & 1.75 & 8 \\
160 & $1.59 \mathrm{E}-03$ & 0.99 & 3 & $1.04 \mathrm{E}-06$ & 2.81 & 33 & $4.27 \mathrm{E}-05$ & 1.87 & 8 \\
320 & $8.02 \mathrm{E}-04$ & 0.99 & 3 & $1.44 \mathrm{E}-07$ & 2.83 & 43 & $1.11 \mathrm{E}-05$ & 1.94 & 7 \\
640 & $4.01 \mathrm{E}-04$ & 1.00 & 3 & $1.99 \mathrm{E}-08$ & 2.85 & 63 & $2.82 \mathrm{E}-06$ & 1.97 & 7 \\
\hline
\end{tabular}

Table 1: Comparison of three fast sweeping methods ( $\Gamma$ is a circle). The error is $\|\phi-d\|_{L^{1}(\Omega)}$. "coc" stands for computed order of convergence, and we report the total number of iterations of the fast sweeping algorithm $N_{i t}$ : 1 fast sweeping iteration corresponds to 1 sweep in each direction.

This method fs2 is only second order accurate, since it consists in the resolution of

$$
\max \left(s D_{x}^{-} \phi_{i, j} ;-s D_{x}^{+} \phi_{i, j} ; 0\right)^{2}+\max \left(s D_{y}^{-} \phi_{i, j} ;-s D_{y}^{+} \phi_{i, j} ; 0\right)^{2}=1,
$$

where $D_{x}^{ \pm} \phi_{i, j}$ and $D_{y}^{ \pm} \phi_{i, j}$ are defined with a decentered second order finite differences formula i.e.,

$$
\begin{aligned}
D_{x}^{-} \phi_{i, j} & =\frac{3 \phi_{i, j}-4 \phi_{i-1, j}+\phi_{i-2, j}}{2 \Delta x}, & D_{y}^{-} \phi_{i, j} & =\frac{3 \phi_{i, j}-4 \phi_{i, j-1}+\phi_{i, j-2}}{2 \Delta y}, \\
D_{x}^{+} \phi_{i, j} & =\frac{-3 \phi_{i, j}+4 \phi_{i+1, j}-\phi_{i+2, j}}{2 \Delta x}, & D_{y}^{+} \phi_{i, j} & =\frac{-3 \phi_{i, j}+4 \phi_{i, j+1}+\phi_{i, j+2}}{2 \Delta y} .
\end{aligned}
$$

Methods fs3Z and fs2 are not based on the same idea, and it is not clear whether a third order method built similarly to $\mathrm{fs} 2$ is tractable or not, although similar methods have been used in the context of fast marching algorithms [19,4]. The three methods fs1, fs2 and fs3Z can be written in the form (3.6). Unlike $\mathrm{fs} 1$ and $\mathrm{fs} 2$, the computation of $\phi_{\min }^{x}$ and $\phi_{\min }^{y}$ in $\mathrm{fs} 3 \mathrm{Z}$ requires the current value $\phi_{i, j}$. This kind of relaxation effect might explain why the number of iterations increases when $h$ decreases in $\mathrm{fs} 3 \mathrm{Z}$, while it remains constant for $\mathrm{fs} 1$ and $\mathrm{fs} 2$.

We illustrate the performances of this second order fast sweeping on the following example: $\Omega$ is the square $[-1 ; 1]$, and $\Gamma$ is the circle with center $(0,0)$ and radius 0.5 . We initialize the fast sweeping algorithm by setting the distance function in the narrow band $B_{n}$, and we recall that these values remain fixed during the computation. We compare the results obtained with the methods fs1, fs3Z and fs2. Note that for second and third order methods, we first apply fs1 in order to have a suitable initial state. We compute the $L^{1}$ error on $\phi$ on the whole domain $\Omega$ and show the results in Table 1.

As expected, this method almost reaches a globally second order accurate. Compared to the high order method of [23], the number of iterations needed to achieve convergence is relatively small and stable, i.e., does not depend on the mesh size. Since the second order scheme is not very expensive compared to the first order, it will be handful to quickly (and rather accurately) compute the distance function in regions far enough from the interface.

\subsection{Mixed method}

The main goal we want to achieve is the construction of a fast method to solve the eikonal equation, with high order accuracy in the vicinity of the interface, where geometric properties are of interest.

We have showed that the relaxation algorithm may be very accurate near the interface, but it is slower than the fast sweeping approach. However, the fast sweeping method requires a good initial guess near the interface. It is then natural to couple the two methods in the following way:

- Initialization: we use the relaxation method to fix the values of $\phi$ in the narrow band $B_{n}$,

- Fast sweeping: we use the second order fast sweeping method to compute the distance function far from the interface. 


\begin{tabular}{|c|c|c|c|c|c|c|c|c|c|c|}
\hline \multirow{2}{*}{$\frac{1}{h}$} & \multicolumn{2}{|c|}{$\left\|\phi_{h}-d\right\|_{L^{1}(\Omega)}$} & \multicolumn{2}{|c|}{$\left\|\phi_{h}-d\right\|_{L^{\infty}\left(B_{n}\right)}$} & \multicolumn{2}{|c|}{$\left\|\kappa_{h}-\kappa\right\|_{L^{\infty}(\Gamma)}$} & \multicolumn{2}{|c|}{$\left\|\kappa_{h}-\kappa\right\|_{L^{\infty}(\Gamma)}$} & \multirow{2}{*}{$N_{i t}$} & \multirow{2}{*}{ CPU time } \\
\hline & err & $\mathrm{coc}$ & err & $\operatorname{coc}$ & err & $\mathrm{coc}$ & err & $\mathrm{coc}$ & & \\
\hline 20 & $3.37 \mathrm{E}-03$ & - & $3.12 \mathrm{E}-02$ & - & $1.81 \mathrm{E}-02$ & & $8.95 \mathrm{E}-02$ & - & 24 & 0.048 \\
\hline 40 & $4.25 \mathrm{E}-04$ & 2.88 & $1.51 \mathrm{E}-03$ & 4.22 & $5.15 \mathrm{E}-03$ & 1.75 & $4.01 \mathrm{E}-02$ & 1.12 & 26 & 0.1 \\
\hline 80 & $1.10 \mathrm{E}-04$ & 1.92 & $2.48 \mathrm{E}-04$ & 2.56 & $1.28 \mathrm{E}-03$ & 1.98 & $1.91 \mathrm{E}-02$ & 1.05 & 28 & 0.604 \\
\hline 160 & $3.19 \mathrm{E}-05$ & 1.77 & $2.85 \mathrm{E}-05$ & 3.09 & $4.65 \mathrm{E}-04$ & 1.44 & $9.38 \mathrm{E}-03$ & 1.01 & 31 & 2.560 \\
\hline 320 & $9.43 \mathrm{E}-06$ & 1.75 & $3.37 \mathrm{E}-06$ & 3.06 & $1.60 \mathrm{E}-04$ & 1.53 & $4.52 \mathrm{E}-03$ & 1.05 & 34 & 11.09 \\
\hline 640 & $2.57 \mathrm{E}-06$ & 1.87 & $6.09 \mathrm{E}-07$ & 2.46 & $1.97 \mathrm{E}-04$ & -0.30 & $2.34 \mathrm{E}-03$ & 0.95 & 36 & 46.88 \\
\hline 1280 & $6.82 \mathrm{E}-07$ & 1.91 & $6.98 \mathrm{E}-08$ & 3.12 & $1.51 \mathrm{E}-04$ & 0.38 & $1.18 \mathrm{E}-03$ & 0.98 & 38 & 206.1 \\
\hline
\end{tabular}

Table 2: Convergence errors for Example 1 (circle). Errors on the curvature are relative errors. $N_{i t}$ stands for the total number of iterations required by the reinitialization algorithm (relaxation + fast sweeping).

In order to get a good approximation of the distance function near the interface during the initialization step, we only need to accurately solve (3.3) in the vicinity of the interface, i.e., on $B_{n}$. The relaxation method has been shown to be third-order accurate near the interface [6], so we stop the computation when

$$
\left\|\phi^{(n+1)}-\phi^{(n)}\right\|_{L^{1}\left(B_{n}\right)} \leq(\Delta x)^{3} .
$$

Assuming uniformly distributed errors, this stopping criterion ensures that the error on $\phi$ in the band $B_{n}$ is of order 3 , thus that the curvature is computed with at least first order accuracy. Using an explicit Euler method for the time discretization of (3.3), we infer that the stopping criterion (3.7) is equivalent to requiring

$$
\left\|\left|\nabla \phi^{n}\right|-1\right\|_{L^{1}\left(B_{n}\right)} \leq(\Delta x)^{2} .
$$

Numerical simulations (not shown here) have been performed with both criteria (3.7) and (3.8) and give comparable results.

\subsection{Numerical illustrations}

We illustrate the behaviour of the mixed method for reinitialization on two cases: a closed interface and an interface crossing the boundary of $\Gamma$.

Example 1: closed interface. The setting is given by (2.7)-(2.10), but with a different radius $R=0.5$. This is a slight modification of the test case of [17] where $\Gamma$ is an ellipse. We choose a circle so as to have exact solutions for the distance function and the curvature.

The results of the mixed reinitialization algorithm are presented in Table 2 . We illustrate the second order accuracy of the method, using the $L^{1}$ norm over the whole domain $\Omega$. This second order is due to the second order fast sweeping step. We compute the $L^{\infty}$ error on $\phi$ in the narrow band $B_{n}$. We observe the expected third order. We show two series of results for the $L^{\infty}$ error on $\kappa$ on the interface. The first one uses the second order finite differences formulas (2.11)-(2.13). The second ones uses a first order formula for the approximation of $\kappa$. The latter exhibits a clear first order convergence, whereas it is not that clear for the usual computation of $\kappa$. However, a linear regression on the results gives an average rate of convergence of 0.996. Even if the error seems to deteriorate for fine meshes, it is still better to use the second order centered finite difference formula since the amplitude of the error is reduced by a factor of at least 10 for reasonable meshes.

Example 2: wave-like interface. Now we turn our attention to a case where $\Gamma$ crosses the boundary of $\Omega$. We use $\Omega=[-1 ; 1]^{2}$ and the interface

$$
\Gamma:=\left\{\left(x-x_{0}\right)^{2}+\left(y-y_{0}\right)^{2}=r_{0}^{2}\right\}
$$



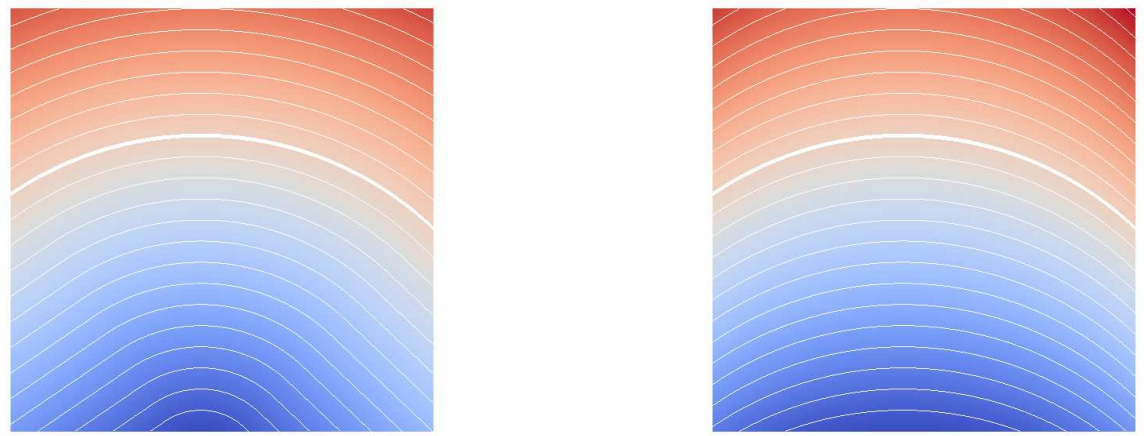

Figure 3: Left: distance function associated to the "wave-like" interface (Example 2). Right: initial level set. The original interface $\Gamma_{0}$ is the thick line. Other lines are isolines of $\phi$.

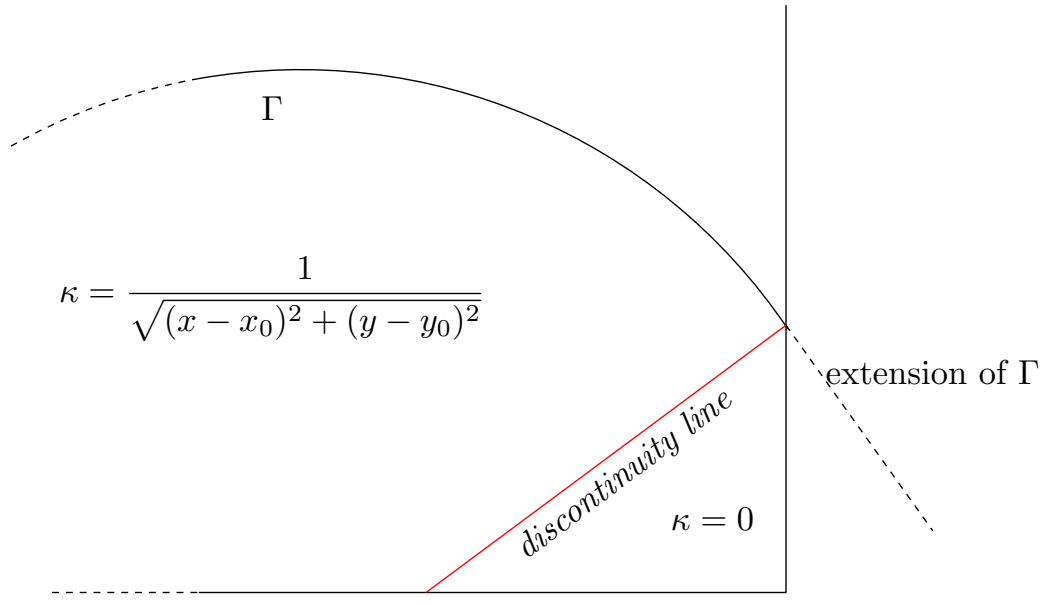

Figure 4: The extension of $\phi$ introduces the red line as a discontinuity line for $D^{2} d$ (hence also for $\kappa)$. Here, $\Gamma$ is part of the circle $\left(x-x_{0}\right)^{2}+\left(y-y_{0}\right)^{2}=r_{0}^{2}$ and crosses the boundary of $\Omega$.

with $\left(x_{0}, y_{0}\right)=(-0.1 ;-1.2)$ and $r_{0}=1.7$, see Figure $3 . \Gamma$ is the graph of a $\mathcal{C}^{1}$ function and we start with

$$
\phi_{0}(x, y)=y-y_{0}-\sqrt{r_{0}^{2}-\left(x-x_{0}\right)^{2}} .
$$

The distance function we want to compute is depicted in Figure 3.

Note that when the interface touches the boundary of the domain, we need to extend $\Gamma$ outside $\Omega$. For this particular case, one could choose the natural extension by the circle $\mathcal{C}_{0}$ with center $\left(x_{0}, y_{0}\right)$ and radius $r_{0}$. In order to have a method that can be easily adapted for any kind of interfaces, we choose an extension with straight lines, tangent to $\Gamma$. Thus, $\phi_{0}$ is the distance to $\mathcal{C}_{0}$ except in the bottom left and the bottom right corners.

With this kind of extension, we may encounter inflows for the gradient of the level set. Thus we have to handle part of the boundary using Dirichlet type boundary conditions. The main difference with usual Dirichlet boundary conditions is that the boundary values we want to enforce are $a$ priori unknown. Using linear extensions allows to accurately know the boundary values, so that ideas from [10] might be considered, i.e., the use of Richardson extrapolation or a Lax-Wendroff procedure. However, the extension introduces a discontinuity line for $D^{2} \phi$ (cf. Figure 4), so that none of these ideas prevents the loss of the third order accuracy in a neighbourhood of $\Gamma$. Thus we choose a more naive approach to enforce Dirichlet conditions: 


\begin{tabular}{|c|c|c|c|c|c|c|c|c|c|c|}
\hline \multirow{2}{*}{$\frac{1}{h}$} & \multirow{2}{*}{$\frac{\| \phi_{h}-d \mid}{\mathrm{err}}$} & \multirow{2}{*}{$\begin{array}{l}L^{1}(\Omega) \\
\operatorname{coc}\end{array}$} & \multicolumn{2}{|c|}{$\left\|\phi_{h}-d\right\|_{L^{\infty}\left(B_{n}\right)}$} & \multicolumn{2}{|c|}{$\left\|\kappa_{h}-\kappa\right\|_{L^{1}(\Gamma)}$} & \multirow{2}{*}{$\frac{\| \kappa_{h}-\kappa}{\text { err }}$} & \multirow{2}{*}{$\begin{array}{r}L^{\infty}(\Gamma) \\
\operatorname{coc}\end{array}$} & \multirow{2}{*}{$N_{i t}$} & \multirow{2}{*}{ CPU time } \\
\hline & & & err & $\mathrm{coc}$ & err & $\mathrm{coc}$ & & & & \\
\hline 40 & $4.84 \mathrm{E}-04$ & - & $4.40 \mathrm{E}-03$ & - & 7.53E-03 & - & $8.36 \mathrm{E}-02$ & - & 19 & 0.143 \\
\hline 80 & $5.50 \mathrm{E}-05$ & 3.08 & $3.40 \mathrm{E}-04$ & 3.63 & $3.44 \mathrm{E}-03$ & 1.11 & $8.44 \mathrm{E}-02$ & -0.01 & 23 & 0.599 \\
\hline 160 & $9.01 \mathrm{E}-06$ & 2.59 & $3.94 \mathrm{E}-05$ & 3.08 & $2.38 \mathrm{E}-03$ & 0.53 & 8.99E-02 & -0.09 & 25 & 2.468 \\
\hline 320 & $2.11 \mathrm{E}-06$ & 2.09 & $4.84 \mathrm{E}-06$ & 3.01 & $1.14 \mathrm{E}-03$ & 1.05 & $1.06 \mathrm{E}-01$ & -0.24 & 28 & 10.51 \\
\hline 640 & $5.32 \mathrm{E}-07$ & 1.98 & $9.54 \mathrm{E}-07$ & 2.34 & $6.21 \mathrm{E}-04$ & 0.87 & $8.44 \mathrm{E}-02$ & 0.33 & 30 & 43.79 \\
\hline 1280 & $1.40 \mathrm{E}-07$ & 1.93 & $2.55 \mathrm{E}-07$ & 1.90 & $3.14 \mathrm{E}-04$ & 0.98 & $5.96 \mathrm{E}-02$ & 0.50 & 33 & 186.6 \\
\hline
\end{tabular}

Table 3: Convergence errors for Example 2.

- relaxation step: at the inflow boundary only, we add a Dirichlet step where we replace $\phi$ by the distance to the extension of $\Gamma$,

- fast sweeping step: the conditions are enforced in a band near the inflow boundary, and not only on the boundary.

In our case, the bottom left and bottom right parts are Dirichlet boundaries, more precisely, denoting $\partial \Omega_{D}$ the inflow boundary, we have

$$
\partial \Omega_{D}=\left\{(x, y) /\left(x=x_{\min } \text { or } x=x_{\max }\right) \text { and } y \leq y_{0}+\sqrt{r_{0}^{2}-\left(x-x_{0}\right)^{2}}\right\} .
$$

We present the results in Table 3. Again, the global second order accuracy is reached. The linear extension of $\Gamma$ introduces shocks near the intersection between $\Gamma$ and the boundary of $\Omega$; this leads to a loss of accuracy in $B_{n}$. As a result, the $L^{\infty}$ error on $\phi$ is only second order accurate and the computation of the curvature is also altered. We do not have the first order accuracy in the $L^{\infty}$ norm on $\Gamma$, but we still have it for the $L^{1}$ norm. This implies that $\kappa$ is well computed away from the boundary points only. Note also that the number of iterations needed for convergence is different than for the previous example, but it is still increasing very slowly. As a comparison, for both examples, the resolution of the eikonal equation using only the relaxation method would have required more than 2000 iterations on the finest mesh (and then much more CPU time).

\section{Coupling transport and reinitialization}

In this part, we investigate the coupling between the transport of the interface and the computation of the distance function. We present results where the interface is passively advected by a velocity field. We choose divergence free velocity fields to emulate an incompressible flow. The challenge is to find a strategy that avoids too many reinitialization steps, since this procedure may introduce some errors in the position of the interface.

\subsection{Advection}

Recall that we have at hand a velocity field defined on $\Omega$. In some applications, a different velocity is used for the advection step. This so called extension velocity is supposed to keep $\phi$ as close as possible to the signed distance function and help reducing the number of reinitialization steps. However, distortions of $\Gamma$ might lead to a loss of smoothness, i.e., the original velocity might be smoother than the extension velocity, and we infer that it could lead to higher errors during the advection part. We choose to work with the original velocity, and to solve

$$
\partial_{t} \phi+\boldsymbol{U} \cdot \nabla \phi=0
$$

Note that we use (4.1) even in the cases where $\boldsymbol{U}$ is divergence free. A WENO-5 scheme is used to compute spatial derivatives, and the time evolution is done with a RK3-TVD scheme. For this 
advection, we use a time step that satisfies the CFL condition

$$
\Delta t<\frac{\Delta x}{\max |\boldsymbol{U}|} .
$$

\subsection{Number of reinitialization steps}

In the usual level set strategies, it is claimed that reinitialization is necessary, but should not be performed too many times. This is again due to the slight displacement of the interface induced by the reinitialization algorithms. Even with a high order reinitialization, the computation of the signed distance function at each time step might lead to significant errors in the position of the interface for long times. It is then necessary to limit the number of reinitialization steps. For a given final time, one might think of setting a maximum number of reinitialization steps, and distribute them uniformly in time. However, it is unclear how to choose the maximum number of reinitialization steps. Our approach is based on the fact that, during the transport of $\Gamma, \phi$ slowly deviate from a distance function. We choose to perform the reinitialization when $\phi$ is "too far away" from the signed distance function.

\subsection{Strategy}

Recalling that the distance function has the property $|\nabla d|=1$, we introduce the quantity $r_{g}(\nabla \phi):=|| \nabla \phi \mid-1 \|_{L^{1}(\Omega)}$. We choose a threshold $\delta>0$ and we consider the following continuous problem:

- Initialization: start with $\phi_{0}=d_{0}$, the signed distance function to $\Gamma_{0}$,

- Transport: while $r_{g}(\nabla \phi)<\delta$, evolve $\phi$ according to (4.1),

- Reinitialization: when $r_{g}(\nabla \phi)=\delta$, set $\phi_{0}=\phi$ and recompute the signed distance function $d$. Set $\phi=d$ and go to Transport

The method we want to use is based on a discrete approximation of this problem. The only modification is that the reinitialization steps occur when $r_{g}(\nabla \phi) \geq \delta$. Using the continuous problem as a reference, we see that the solution we want to approximate does not depend on the mesh size or the time step. This is a major difference compared to usual level set strategies where the reinitialization steps are performed every 5 or 10 time iterations and/or the reinitialization procedure is performed only in a narrow band, typically of length $5 \Delta x$, near the interface. It is then reasonable to check if there is a convergence order. Numerical simulations tend to assess the expected high order accuracy.

\subsection{Numerical illustrations}

We illustrate the performances of the new strategy on several examples. We start by showing that the coupling with the transport equation preserves the first order accuracy on $\kappa$. This is not the case for usual level set strategies, and the results obtained with the new strategy compare well with standard approaches. We then illustrate the necessity of the reinitialization procedure when steep gradients are likely to be created. We also investigate the influence of the threshold $\delta$ in the scheme, and we end the section by showing that the method depends very weakly on the time step, which makes it robust if coupled with problems where $\Delta t$ is modified during the simulation.

\subsubsection{Closed interface and vortex}

We first present some results on a moving closed interface.

Example 3: vortex case. This example is a slight modification of the original vortex example of [1]. The difference is that we impose a periodic velocity so as to recover the initial interface 


\begin{tabular}{|c||c|c||c|c||c|c||c||c|c|}
\hline \multirow{2}{*}{\multicolumn{1}{|c||}{$\frac{\|}{h}$}} & $\left\|\phi_{h}-d\right\|_{L^{1}(\Omega)}$ & $\left\|\phi_{h}-d\right\|_{L^{\infty}\left(B_{n}\right)}$ & $\left\|\kappa_{h}-\kappa\right\|_{L^{\infty}(\Gamma)}(t=4)$ & \multirow{2}{*}{$N_{\text {red }}$} & \multicolumn{2}{|c|}{$\left\|\kappa_{h}-\kappa\right\|_{L^{\infty}(\Gamma)}(t=2)$} \\
\cline { 2 - 6 } \cline { 5 - 6 } & val & coc & val & coc & val & coc & & val & coc \\
\hline 40 & $8.62 \mathrm{E}-04$ & - & $2.44 \mathrm{E}-03$ & - & $2.11 \mathrm{E}-01$ & - & 17 & $1.47 \mathrm{E}-01$ & - \\
80 & $8.60 \mathrm{E}-05$ & 3.27 & $3.04 \mathrm{E}-04$ & 2.95 & $5.59 \mathrm{E}-02$ & 1.88 & 17 & $4.63 \mathrm{E}-02$ & 1.64 \\
160 & $3.08 \mathrm{E}-05$ & 1.47 & $2.23 \mathrm{E}-05$ & 3.73 & $1.41 \mathrm{E}-02$ & 1.97 & 16 & $1.51 \mathrm{E}-02$ & 1.60 \\
320 & $9.30 \mathrm{E}-06$ & 1.72 & $1.97 \mathrm{E}-06$ & 3.48 & $5.22 \mathrm{E}-03$ & 1.43 & 16 & $4.60 \mathrm{E}-03$ & 1.70 \\
640 & $2.52 \mathrm{E}-06$ & 1.88 & $1.15 \mathrm{E}-07$ & 4.09 & $3.65 \mathrm{E}-04$ & 3.83 & 17 & $3.16 \mathrm{E}-04$ & 3.86 \\
1280 & $6.59 \mathrm{E}-07$ & 1.94 & $1.07 \mathrm{E}-08$ & 3.43 & $9.73 \mathrm{E}-05$ & 1.91 & 16 & $2.17 \mathrm{E}-04$ & 0.54 \\
\hline
\end{tabular}

Table 4: Example 3 (vortex case). Errors on $\phi$ and $\kappa$ at $t=4$ (first three columns). Total number of reinitialization steps $\left(N_{\text {red }}\right)$. Error on $\kappa$ at $t=2$ (last column).

after a given time. The computational domain is $\Omega=[0,1]^{2}$. The initial interface $\Gamma_{0}$ is the circle with center $\left(\frac{1}{2}, \frac{3}{4}\right)$ and radius 0.15 . The velocity field is given by the scalar potential :

$$
\boldsymbol{U}=\cos \left(\frac{\pi t}{T}\right) \nabla^{\perp} \omega, \quad \omega=\frac{1}{2 \pi} \sin ^{2}(\pi x) \sin ^{2}(\pi y),
$$

with $T=2$. With this choice of $\boldsymbol{U}$, the interface is $\Gamma=\Gamma_{0}$ for $t=0, t=2$ and $t=4$. The initial level set is the signed distance to $\Gamma_{0}$. We use meshes with typical length from $1 / 40$ to $1 / 1280$, and perform simulations for $t \in[0,4]$. We use the threshold $\delta=0.1$ for reinitialization. The computation of the signed distance is enforced at $t=4$ for each mesh. We report the results in Table 4 at $t=4$, as well as the error on $\kappa$ at $t=2$. We also report the total number of reinitializations steps during the whole computation.

These results confirm the good behaviour of the method, even when coupled with the transport of the interface. As expected, the number of reinitialization steps needed is almost uniform in $h$, since the strategy is based on the approximation of the same continuous problem.

The results on $\kappa$ at $t=2$ are presented since it is not a reinitialization time, but $\Gamma$ is the original circle. Since we enforce the computation of the signed distance function at $t=4$, it is natural to wonder whether the good approximations properties that we observe is due to that last reinitialization or not. Results at $t=2$ confirm that we have the first order accuracy on $\kappa$, even for intermediate times.

Note that for this example, we get better results than the third order accuracy expected for the $L^{\infty}$ error on $\phi$ in the narrow band. This behaviour explains the higher order for the curvature.

\subsubsection{Comparison with usual level set strategies}

When accuracy is needed near the interface, usual level set strategies consists of using the relaxation method. The reinitialization procedure is performed at each time step (or every 5 or 10 time step), and a fixed number of iterations ( 3 or 5 e.g. ) is used. We use the same setting as before, and perform the computation until $t=2$. We compare four cases:

1. 5 iterations of the relaxation method, every 5 time step,

2. 3 iterations of the relaxation method at every time step,

3. our new method, with $\delta=0.1$,

4. our new method, with $\delta=0.01$.

Results are presented in tables 5 and 6 . We compare the computation of the curvature at $t=2$, the position of the interface and the volume loss. Since $\nabla \cdot \boldsymbol{U}=0$, the volume should be constant, i.e.,

$$
\frac{d}{d t} \int_{\{\phi(\boldsymbol{x}) \leq 0\}} 1 d \boldsymbol{x}=0 .
$$




\begin{tabular}{|c||c|c||c|c||c|c||c|c|}
\hline \multicolumn{1}{|c||}{} & \multicolumn{2}{c||}{ case 1} & \multicolumn{2}{c||}{ case 2} & \multicolumn{2}{c||}{ case 3} & \multicolumn{2}{c|}{ case 4} \\
\cline { 2 - 8 }$h$ & err. & coc & err. & coc & err. & coc & err. & coc \\
\hline 40 & $1.82 \mathrm{E}-01$ & - & $1.18 \mathrm{E}-01$ & - & $1.47 \mathrm{E}-01$ & - & $1.22 \mathrm{E}-01$ & - \\
80 & $5.53 \mathrm{E}-02$ & 1.69 & $6.35 \mathrm{E}-02$ & 0.87 & $4.63 \mathrm{E}-02$ & 1.64 & $4.16 \mathrm{E}-02$ & 1.53 \\
160 & $7.07 \mathrm{E}-02$ & -0.35 & $7.62 \mathrm{E}-02$ & -0.26 & $1.51 \mathrm{E}-02$ & 1.61 & $1.30 \mathrm{E}-02$ & 1.66 \\
320 & $6.56 \mathrm{E}-02$ & 0.11 & $9.56 \mathrm{E}-02$ & -0.33 & $4.58 \mathrm{E}-03$ & 1.71 & $3.87 \mathrm{E}-03$ & 1.74 \\
640 & $1.10 \mathrm{E}-01$ & -0.75 & $2.01 \mathrm{E}-01$ & -1.07 & $3.27 \mathrm{E}-04$ & 3.80 & $3.05 \mathrm{E}-04$ & 3.66 \\
\hline
\end{tabular}

Table 5: $L^{\infty}$ error on the curvature at $t=2$ for the 4 cases

\begin{tabular}{|c||c|c||c|c||c|c||c|c|}
\hline \multicolumn{1}{|c||}{} & \multicolumn{2}{c||}{ case 1} & \multicolumn{2}{c||}{ case 2} & \multicolumn{2}{c||}{ case 3} & \multicolumn{2}{c|}{ case 4} \\
\cline { 2 - 8 } & pos. & vol. loss & pos. & vol. loss & pos. & vol. loss & pos. & vol. loss \\
\hline 40 & $2.19 \mathrm{E}-03$ & $6.82 \mathrm{E}-03$ & $7.89 \mathrm{E}-04$ & $1.06 \mathrm{E}-03$ & $2.20 \mathrm{E}-03$ & $5.67 \mathrm{E}-03$ & $1.03 \mathrm{E}-03$ & $2.50 \mathrm{E}-03$ \\
80 & $2.99 \mathrm{E}-04$ & $9.39 \mathrm{E}-04$ & $1.58 \mathrm{E}-04$ & $2.72 \mathrm{E}-04$ & $2.90 \mathrm{E}-04$ & $4.06 \mathrm{E}-04$ & $1.77 \mathrm{E}-04$ & $1.53 \mathrm{E}-04$ \\
160 & $6.45 \mathrm{E}-05$ & $1.86 \mathrm{E}-04$ & $9.20 \mathrm{E}-05$ & $2.19 \mathrm{E}-04$ & $2.07 \mathrm{E}-05$ & $1.88 \mathrm{E}-05$ & $1.80 \mathrm{E}-05$ & $1.30 \mathrm{E}-05$ \\
320 & $3.19 \mathrm{E}-05$ & $7.38 \mathrm{E}-05$ & $4.27 \mathrm{E}-05$ & $1.15 \mathrm{E}-04$ & $1.59 \mathrm{E}-06$ & $9.66 \mathrm{E}-07$ & $1.44 \mathrm{E}-06$ & $9.25 \mathrm{E}-07$ \\
640 & $1.63 \mathrm{E}-05$ & $3.66 \mathrm{E}-05$ & $2.25 \mathrm{E}-05$ & $6.21 \mathrm{E}-05$ & $7.83 \mathrm{E}-08$ & $4.30 \mathrm{E}-08$ & $8.02 \mathrm{E}-08$ & $3.50 \mathrm{E}-08$ \\
\hline
\end{tabular}

Table 6: Error on the position of $\Gamma$ and volume loss at $t=2$.

The total number of reinitialization steps for the finest mesh is respectively 256, 1240, 10 and 86 for each case. The results show that usual reinitialization strategies do not ensure an accurate computation of $\kappa$, while the new method is first order accurate on the curvature. We also notice that both the position of $\Gamma$ and the volume are computed more accurately with the new method. For cases (1) and (2), they are approximated with first order accuracy, whereas the new method exhibits an average rate of convergence of almost 4 .

The maximum deformation of $\Gamma$ is for $t=1$. In this example, the distortion is not too severe, so that cases (3) and (4) (i.e., the new method with $\delta=0.1$ and $\delta=0.01$ ) give similar results. Table 5 indicates that a rather small number of reinitialization steps is needed to obtain accurate results. In this particular case, it is even better to use the transport equation without any reinitialization ( $c f$. , also 4.4.4). However, as shown in the next section, reinitialization steps are sometimes necessary to obtain accurate approximations.

\subsubsection{Formation of steep gradients near the interface}

On the previous example, since the distortion of $\Gamma$ is not too severe, there is no creation of (very) steep gradients near the interface. Reinitialization might be critical to accurately compute the curvature when the flow tends to create steep gradients, for instance in the case of the merging of two bubbles [20]. We illustrate this fact and the behaviour of our method on two cases.

Example 4: We use the following setting:

$$
\begin{aligned}
\Omega & =[-1 ; 1]^{2}, \\
\Gamma_{0} & =\{y=0\}, \\
\boldsymbol{U} & =\nabla^{\perp} \omega, \\
\omega(x, y) & =\frac{1}{2 \pi} \sin (\pi x)^{2} \sin (\pi y)^{2} .
\end{aligned}
$$

With this choice, the interface does not move, i.e., $\Gamma=\Gamma_{0}$. The final time is $t=6$. Without reinitialization, the isocontours of $\phi$ are greatly distorted, and steep gradients appear, even in the neighbourhood of $\Gamma, c f$. , Figure 5 . 

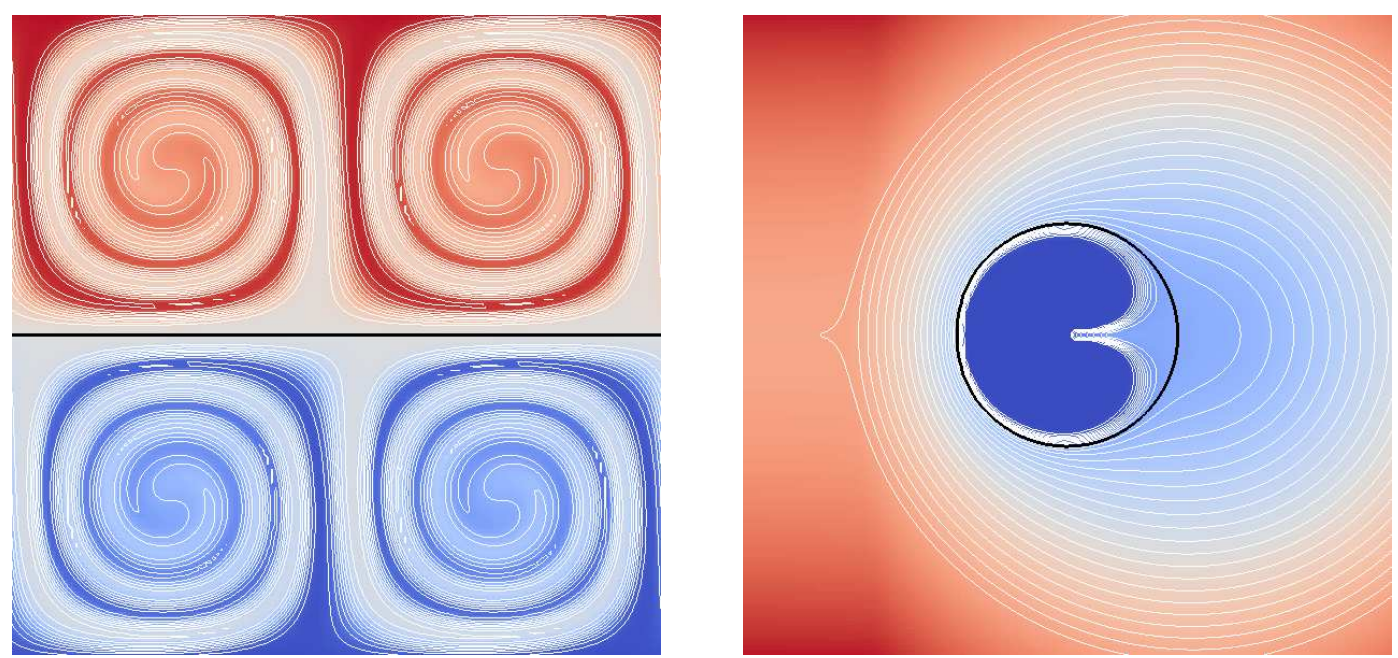

Figure 5: Isocontours of $\phi$ for example 4 (left) and example 5 (right) at $t=6$ without reinitialization. $\Gamma$ is the dark thick line.

\begin{tabular}{|c||c|c||c|c||c|c|}
\hline \multicolumn{1}{|c||}{$\frac{1}{h}$} & \multicolumn{2}{c||}{$\delta=0.01$} & \multicolumn{2}{c||}{$\delta=0.1$} & \multicolumn{2}{c|}{$\delta=1}$. \\
\cline { 2 - 7 } & err. & coc & err. & coc & err. & coc \\
\hline 40 & $1.03 \mathrm{E}-02$ & - & $1.65 \mathrm{E}-02$ & - & $7.20 \mathrm{E}+00$ & - \\
80 & $2.68 \mathrm{E}-03$ & 1.90 & $1.10 \mathrm{E}-03$ & 3.84 & $4.11 \mathrm{E}-01$ & 4.06 \\
160 & $4.13 \mathrm{E}-04$ & 2.68 & $1.54 \mathrm{E}-04$ & 2.81 & $1.55 \mathrm{E}-02$ & 4.69 \\
320 & $1.50 \mathrm{E}-04$ & 1.46 & $6.70 \mathrm{E}-05$ & 1.20 & $8.37 \mathrm{E}-04$ & 4.19 \\
640 & $3.59 \mathrm{E}-05$ & 2.05 & $5.30 \mathrm{E}-05$ & 0.34 & $5.04 \mathrm{E}-05$ & 4.04 \\
\hline
\end{tabular}

Table 7: Example $4:\left\|\kappa_{h}-\kappa\right\|_{L^{\infty}(\Gamma)}$ at $t=6$ and computed order of convergence. The number of reinitialization steps for the last mesh is 175,40 and 1 respectively.

Example 5: : flow around a circular cylinder. In the case of a flow around a motionless cylinder, the usual form for the components of the velocity is given in polar coordinates by

$$
\begin{aligned}
& V_{r}=\left(U_{\infty}-\frac{K}{r^{2}}\right) \cos (\theta), \\
& V_{\theta}=-\left(U_{\infty}+\frac{K}{r^{2}}\right) \sin (\theta) .
\end{aligned}
$$

We choose $U_{\infty}=1, K=1, \Gamma_{0}$ is the circle with radius 1 , centered at the origin, and the numerical domain is $\Omega=[-3 ; 3]^{2}$. We slightly change the velocity field inside the cylinder to avoid the singularity at the origin, by setting

$$
U_{r}=\alpha c(r) V_{r}, \quad U_{\theta}=\alpha c(r) V_{\theta},
$$

where $c(r)=\min \left(1, \frac{r}{0.5}\right)^{3}$ and $\alpha$ is chosen so that $\| \boldsymbol{U}_{L^{\infty}(\Omega)}=1$. With this setting, the interface does not move (even if $\boldsymbol{U} \neq 0$ on $\Gamma$ ). Again, without reinitialization, the isocontours of $\phi$ are severely distorted, leading to numerical issues for the transport itself, $c f$., Figure 5 . As for Example 4 , the final time is $t=6$.

For both examples 4 and 5, we compute the curvature at the final time, with three different values of $\delta$. We enforce a reinitialization step at the end. For $\delta=0.01$, the distortion of the isocontours of $\phi$ between two reinitialization steps is mild, whereas for $\delta=1$, there is no reinitialization until the final time. The $L^{\infty}$ error on the curvature (on $\Gamma$ ) is reported in Tables 7 and 8 .

In both cases, results are improved thanks to the reinitialization procedure. In Example 4, a fine mesh $(640 \times 640)$ is necessary to obtain comparable results. In the case of the flow around 


\begin{tabular}{|c||c|c||c|c||c|c|}
\hline \multicolumn{1}{|c||}{\multirow{2}{*}{$\frac{1}{h}$}} & \multicolumn{2}{c||}{$\delta=0.01$} & \multicolumn{2}{c||}{$\delta=0.1$} & \multicolumn{2}{c|}{$\delta=1}$. \\
\cline { 2 - 7 } & err. & coc & err. & coc & err. & coc \\
\hline 40 & $7.24 \mathrm{E}-02$ & - & $8.35 \mathrm{E}-02$ & - & $1.40 \mathrm{E}+00$ & - \\
80 & $3.91 \mathrm{E}-02$ & 0.87 & $1.68 \mathrm{E}-02$ & 2.27 & $2.15 \mathrm{E}+00$ & -0.61 \\
160 & $9.71 \mathrm{E}-03$ & 1.99 & $5.09 \mathrm{E}-03$ & 1.71 & $3.53 \mathrm{E}+00$ & -0.71 \\
320 & $3.66 \mathrm{E}-03$ & 1.40 & $2.51 \mathrm{E}-03$ & 1.01 & $8.22 \mathrm{E}+01$ & -4.52 \\
640 & $2.51 \mathrm{E}-03$ & 0.54 & $1.88 \mathrm{E}-03$ & 0.42 & $1.57 \mathrm{E}+02$ & -0.93 \\
\hline
\end{tabular}

Table 8: Example 5: $\left\|\kappa_{h}-\kappa\right\|_{\mathrm{E}^{\infty}(\Gamma)}$ at $t=6$ and computed order of convergence.
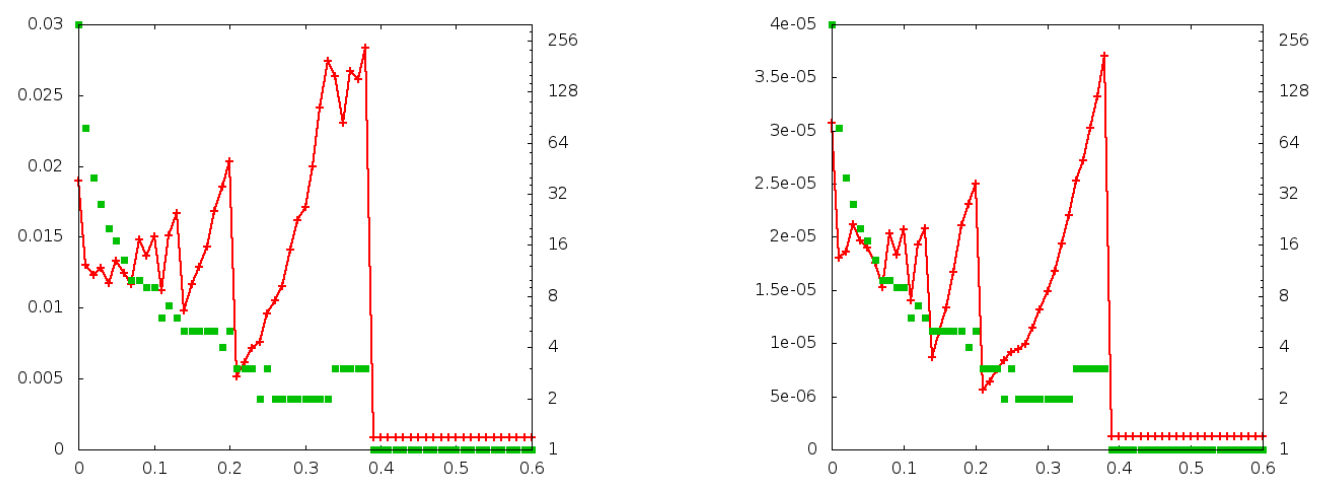

Figure 6: Example 3 (vortex case): Error on the curvature (left) and the position (right) of $\Gamma$, depending on the value of $\delta$. The squares represent the number of reinitialization steps required (logarithmic scale).

a cylinder (example 5), reinitialization is even necessary to get a consistent computation of $\kappa$. The computation of other quantities (not shown here), such as the position of the interface or the volume loss, shows the same behaviour. This example confirms that the reinitialization steps are indeed necessary in some cases to get stable and accurate enough numerical simulations.

\subsubsection{Impact of the threshold $\delta$}

We now compare the results we obtain with several values of the threshold $\delta$ that controls the reinitialization steps. The limit $\delta \rightarrow 0$ corresponds to perform the reinitialization procedure at every time step, for which we expect a loss of accuracy on $\kappa$. The limit $\delta \rightarrow+\infty$ corresponds to the case of transport alone. We test the influence of $\delta$ on two examples: the case given in 4.4.2 and the flow around a cylinder (4.7)-(4.8). The computations are done on a $160 \times 160$ mesh. For each case, we compute the curvature and the position of $\Gamma$ at the final time, respectively $t=2$ and $t=6$.

Results are presented in Figures 6 and 7 respectively. For each case, there seems to be an optimal value of $\delta$ that gives the lowest amplitude on both the curvature and the position of $\Gamma$. The error decreases rapidly when $\delta$ increases, see Figure 8: it confirms that it is best not to choose a too small threshold. This behaviour is more explicit for Example 5: the error on the curvature for $\delta=0$ is almost 0.12 while the minimum error, for $\delta \sim 0.08$, is almost $2.210^{-3}$. For values of $\delta$ that are not too small, there is a range for which the number of reinitializations (and hence the results) are comparable. Finally, in the range of bigger $\delta$, i.e., very small number of reinitialization steps, the behaviour is different in the two examples. In the first case, the slight distortion of $\phi$ and the reversibility of the test case allow better results without reinitialization. On the contrary, in the second case, the distortion is more severe, and increases in time, so that transport alone leads to huge errors compared to computations with reinitializations. In general cases, the behaviour of the isocontours of $\phi$ is not known a priori; the use of a rather small threshold $\delta$ seems to be a good compromise to obtain accurate results. 

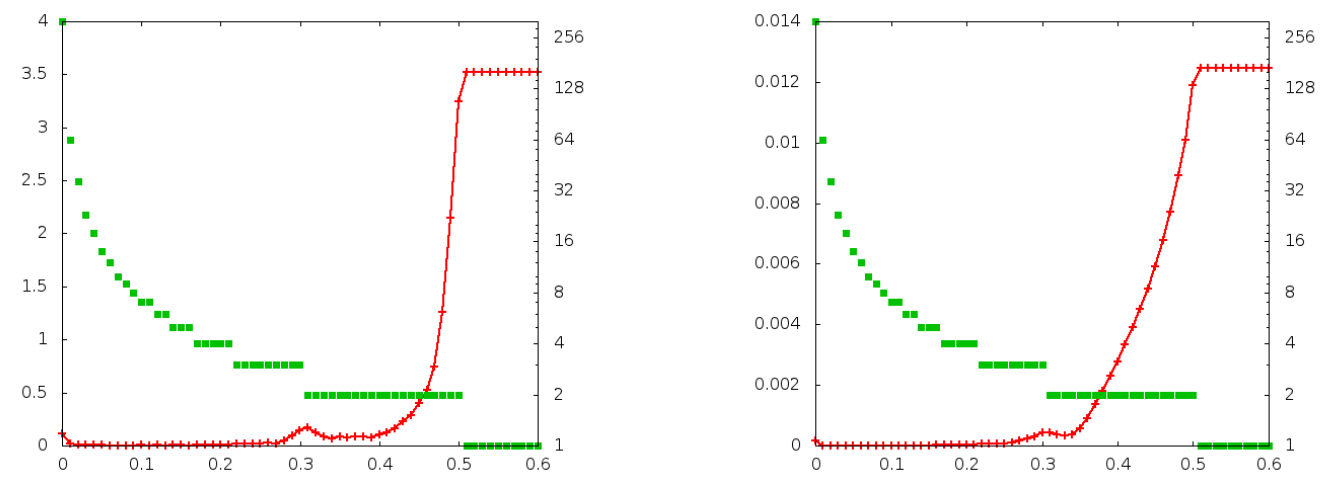

Figure 7: Example 5 (flow around cylinder): Error on the curvature (left) and the position (right) of $\Gamma$, depending on the value of $\delta$. See Figure 8 for a zoom on the region $\delta \in[0 ; 0.3]$. The squares represent the number of reinitialization steps required (logarithmic scale).
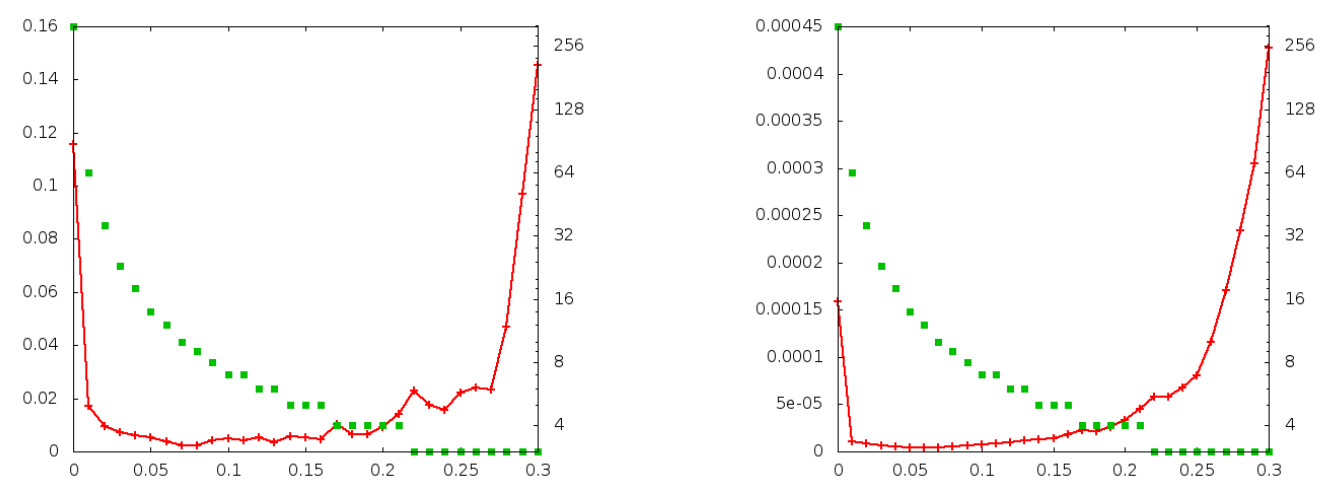

Figure 8: Example 5 (flow around cylinder): Error on the curvature (left) and the position (right) of $\Gamma$, depending on the value of $\delta$. The squares represent the number of reinitialization steps required (logarithmic scale). 


\begin{tabular}{|c||c|c||c|c||c|c||c|c|}
\hline \multicolumn{1}{|c||}{$\Delta t$} & \multicolumn{2}{c||}{$\delta=0$} & \multicolumn{2}{c||}{$\delta=0.01$} & \multicolumn{2}{c||}{$\delta=0.1$} & \multicolumn{2}{c|}{$\delta=1$} \\
\cline { 2 - 8 }$\Delta x$ & err. & $N_{\text {red }}$ & err. & $N_{\text {red }}$ & err. & $N_{\text {red }}$ & err. & $N_{\text {red }}$ \\
\hline 1 & $3.69 \mathrm{E}-01$ & 640 & $3.35 \mathrm{E}-01$ & 180 & $2.96 \mathrm{E}-01$ & 17 & $1.76 \mathrm{E}-02$ & 1 \\
$1 / 2$ & 1.4337 & 1280 & $3.31 \mathrm{E}-01$ & 208 & $2.86 \mathrm{E}-01$ & 17 & $1.76 \mathrm{E}-02$ & 1 \\
$1 / 4$ & 27.158 & 2560 & $3.28 \mathrm{E}-01$ & 220 & $2.82 \mathrm{E}-01$ & 17 & $1.76 \mathrm{E}-02$ & 1 \\
$1 / 8$ & 31.058 & 5120 & $3.69 \mathrm{E}-01$ & 224 & $2.92 \mathrm{E}-01$ & 17 & $1.76 \mathrm{E}-02$ & 1 \\
\hline
\end{tabular}

Table 9: Example 3 with $T=4$. Error $\left\|\kappa_{h}-\kappa\right\|_{L^{\infty}(\Gamma)}$ and total number of reinitialization steps at $t=4$ for several values of $\delta$ et $\Delta t$.

\subsubsection{Sensitivity to the time step}

We have shown that, for rather small values of $\delta$, the numerical method we have presented gives good results compared to usual level set strategies. Recall that in these level set strategies, a reinitialization step is performed every $N$ time steps, where the typical values of $N$ are 1,5 or 10; thus, decreasing $\Delta t$ increases the number of reinitialization steps. It may lead to significant differences in the accuracy. We investigate the behaviour of our new strategy with respect to the time step. The setting is that of Example 3 with $T=4$. Simulations are performed on a $160 \times 160$ mesh, for several values of $\Delta t$. The $L^{\infty}$ error on $\kappa$ at $t=4$ is represented in Table 9 , with different values of $\delta$. Other quantities such as the position of the interface or the volume have been computed (results not shown) and exhibit a similar behaviour. A reinitialization step is enforced at $t=4$.

As expected, the case $\delta=0$ shows the same behaviour as usual level set strategies, i.e., the results get worse and worse as $\Delta t$ decreases. On the contrary, for $\delta>0$, the results weakly depend on $\Delta t$. Thus the method is insensitive to $\Delta t$; in many simulations, it might be useful to change $\Delta t$ according to $\boldsymbol{U}$ to improve computational efficiency. The use of $\delta>0$ ensures that there will be no loss of accuracy.

\section{Discussion}

\subsection{Numerical simulations}

We have described a level set strategy that allows accurate computations of geometric properties of an evolving interface. The strategy is based on a limited number of reinitialization, and may be coupled with any third order accurate method to solve the transport equation. The computational cost is limited thanks to the reinitialization algorithm and the limited number of reinitialization steps needed:

- The cost of one reinitialization step is quite low, but with a good accuracy for the computation of geometric properties. For example, we have shown examples where the total number of iterations during the reinitialization procedure does not exceed 40 , even on a $1280 \times 1280$ mesh, while the relaxation method alone would have required nearly 2000 iterations.

- The number of reinitialization steps is controlled by the distortion of the level set itself, so that reinitialization only occur when flat or steep gradients are created. In other cases, the amplitude of the error on $\kappa$ remain resonable.

Compared to usual level strategies that involves either an incomplete reinitialization and/or a number of reinitialization steps that depends on the mesh size or the time step, this method exhibits a third order accuracy on $\phi$ and then a first order accuracy on $\kappa$. This approximation result allows the coupling between this level set strategy and a numerical scheme involving $\boldsymbol{n}$ or $\kappa$. On a computational point of view, it also allows to anticipate the behaviour of the numerical codes when meshes are refined. These results confirm that it is interesting to have a method for which the total number of reinitialization steps does not depend on $\Delta x$ or $\Delta t$. We performed other simulations with the new reinitialization procedure, but we uniformly distributed (in time) 
reinitialization steps. For the same number of iterations, the results are comparable, yet a little worse on $\kappa$ than the strategy presented in 4.3 .

Although the method is third order accurate for (smooth enough) closed interfaces, the performance is altered when the interface crosses the boundary of the domain. The main issue is that we have artificially introduced a discontinuity by extending $\phi$ with straight lines. The discontinuity line is crossing the interface at the boundary, leading to a loss of accuracy in the computation of geometrical properties (cf. Figure 4). It should be possible to recover the first order accuracy on $\kappa$ in several ways:

- use a different extension for $\Gamma$, to ensure that the regions where the second derivative of $d$ is discontinuous does not touch $\Gamma$,

- use a different stencil to compute $\kappa$ near the boundary, to ensure that only points in the "smooth regions" are involved.

Note that it is sufficient to replace $B_{n}$ (used in the relaxation step) by a neighbourhood of $\Gamma$ containing all the points needed for the computation of geometric properties.

Finally, the method involves a criterion to perform the reinitialization, viz. $r_{g}(\nabla \phi)$, and a threshold $\delta$. We have defined $r_{g}$ on the whole domain $\Omega$, but it is possible to use only a neighbourhood of $\Gamma$, provided it is uniform in $h$. The threshold can be taken as small as desired; decreasing $\delta$ increases the number of reinitialization steps needed. In general, there is no way to know if strong deformations of $\Gamma$ will occur, or if they could be reversible. In such cases, we have shown that it is indeed preferable to use reinitialization, with a threshold that is not too small, in order to get better results. Unfortunately, it remains not clear how to make that choice. In practice, we often use a threshold $\delta \in[0.01 ; 0.1]$, for which there seems to be only a few differences in the results.

\subsection{Extensions of the method}

Although the work presented here is only for $2 D$ cases, it is clear that the same strategy could be carried out in a $3 D$ framework without modifications. We have also chosen methods that should be easily parallelized. In the cartesian framework, the parallelization might be performed using domain decomposition. This should be tractable for the relaxation step, with a small loss of scaling due to the large WENO stencil. If domain decomposition is not efficient enough for the fast sweeping step, the strategy developed by Detrixhe et al.[5] may be used; it is based on the fact that, when sweeping in one direction, nodes in the orthogonal diagonal are independent to one another.

The method has been illustrated on passively advected interfaces with a given velocity field, but it might be used for other applications, provided the transport step is accurate enough. It is suitable in the case of fluid mechanics where the velocity field is available on the whole domain. In the cases where $\boldsymbol{U}$ is only given on $\Gamma$ (eg. for surfaces moving with curvature dependent speed, see [14] e.g. ), it still might be used with a suitable extension velocity construction $[24,15]$.

\section{References}

[1] John B. Bell, Phillip Colella, and Harland M. Glaz. A second-order projection method for the incompressible Navier-Stokes equations. J. Comput. Phys., 85(2):257-283, 1989.

[2] M. Bergmann, J. Hovnanian, and A. Iollo. An accurate cartesian method for incompressible flows with moving boundaries. Commun. Comput. Phys., 15(5):1266-1290, 2014.

[3] J. U. Brackbill, D. B. Kothe, and C. Zemach. A continuum method for modeling surface tension. J. Comput. Phys., 100(2):335-354, 1992.

[4] David L. Chopp. Some improvements of the fast marching method. SIAM J. Sci. Comput., 23(1):230-244 (electronic), 2001. 
[5] Miles Detrixhe, Frédéric Gibou, and Chohong Min. A parallel fast sweeping method for the Eikonal equation. J. Comput. Phys., 237:46-55, 2013.

[6] Antoine du Chéné, Chohong Min, and Frédéric Gibou. Second-order accurate computation of curvatures in a level set framework using novel high-order reinitialization schemes. J. Sci. Comput., 35(2-3):114-131, 2008.

[7] Y. Gorsse, A. Iollo, H. Telib, and L. Weynans. A simple second order cartesian scheme for compressible Euler flows. J. Comput. Phys., 231(23):7780-7794, 2012.

[8] Sigal Gottlieb and Chi-Wang Shu. Total variation diminishing Runge-Kutta schemes. Math. Comp., 67(221):73-85, 1998.

[9] C. W. Hirt, A. A. Amsden, and J. L. Cook. An arbitrary Lagrangian-Eulerian computing method for all flow speeds. Journal of Computational Physics, 14(3):227 - 253, 1974.

[10] Ling Huang, Chi-Wang Shu, and Mengping Zhang. Numerical boundary conditions for the fast sweeping high order WENO methods for solving the eikonal equation. J. Comput. Math., 26(3):336-346, 2008.

[11] Guang-Shan Jiang and Danping Peng. Weighted ENO schemes for Hamilton-Jacobi equations. SIAM J. Sci. Comput., 21(6):2126-2143 (electronic), 2000.

[12] Xu-Dong Liu, Stanley Osher, and Tony Chan. Weighted essentially non-oscillatory schemes. J. Comput. Phys., 115(1):200-212, 1994.

[13] Jacques Magnaudet, Mayela Rivero, and Jean Fabre. Accelerated flows past a rigid sphere or a spherical bubble. I. Steady straining flow. J. Fluid Mech., 284:97-135, 1995.

[14] Stanley Osher and James A. Sethian. Fronts propagating with curvature-dependent speed: algorithms based on Hamilton-Jacobi formulations. J. Comput. Phys., 79(1):12-49, 1988.

[15] Danping Peng, Barry Merriman, Stanley Osher, Hongkai Zhao, and Myungjoo Kang. A PDE-based fast local level set method. J. Comput. Phys., 155(2):410-438, 1999.

[16] Elisabeth Rouy and Agnès Tourin. A viscosity solutions approach to shape-from-shading. SIAM J. Numer. Anal., 29(3):867-884, 1992.

[17] Giovanni Russo and Peter Smereka. A remark on computing distance functions. J. Comput. Phys., 163(1):51-67, 2000.

[18] J. A. Sethian. A fast marching level set method for monotonically advancing fronts. Proc. Nat. Acad. Sci. U.S.A., 93(4):1591-1595, 1996.

[19] James A. Sethian. Fast marching methods. SIAM Rev., 41(2):199-235, 1999.

[20] Mark Sussman, Peter Smereka, and Stanley Osher. A level-set approach for computing solutions to incompressible two-phase flows. Journal of Computational Physics, 114:146-159, 1994.

[21] Yen-Hsi Richard Tsai, Li-Tien Cheng, Stanley Osher, and Hong-Kai Zhao. Fast sweeping algorithms for a class of Hamilton-Jacobi equations. SIAM J. Numer. Anal., 41(2):673-694, 2003.

[22] David E. Womble. A front-tracking method for multiphase free boundary problems. SIAM J. Numer. Anal., 26(2):380-396, 1989.

[23] Yong-Tao Zhang, Hong-Kai Zhao, and Jianliang Qian. High order fast sweeping methods for static Hamilton-Jacobi equations. J. Sci. Comput., 29(1):25-56, 2006. 
[24] Hong-Kai Zhao, T. Chan, B. Merriman, and S. Osher. A variational level set approach to multiphase motion. J. Comput. Phys., 127(1):179-195, 1996.

[25] Hongkai Zhao. A fast sweeping method for eikonal equations. Math. Comp., 74(250):603-627, 2005. 
Centre de recherche INRIA Bordeaux - Sud Ouest

Domaine Universitaire - 351, cours de la Libération - 33405 Talence Cedex (France)

Centre de recherche INRIA Grenoble - Rhône-Alpes : 655, avenue de l'Europe - 38334 Montbonnot Saint-Ismier

Centre de recherche INRIA Lille - Nord Europe : Parc Scientifique de la Haute Borne - 40, avenue Halley - 59650 Villeneuve d'Ascq

Centre de recherche INRIA Nancy - Grand Est : LORIA, Technopôle de Nancy-Brabois - Campus scientifique 615, rue du Jardin Botanique - BP 101 - 54602 Villers-lès-Nancy Cedex

Centre de recherche INRIA Paris - Rocquencourt : Domaine de Voluceau - Rocquencourt - BP 105 - 78153 Le Chesnay Cedex

Centre de recherche INRIA Rennes - Bretagne Atlantique : IRISA, Campus universitaire de Beaulieu - 35042 Rennes Cedex

Centre de recherche INRIA Saclay - Île-de-France : Parc Orsay Université - ZAC des Vignes : 4, rue Jacques Monod - 91893 Orsay Cedex

Centre de recherche INRIA Sophia Antipolis - Méditerranée : 2004, route des Lucioles - BP 93 - 06902 Sophia Antipolis Cedex 